\title{
EMPLEO Y DESEMPLEO EN BAHIA BLANCA EN LOS AÑOS 2003-2013
}

\author{
Gustavo Burachik*
}

recibido: abril 2015 - aceptado: junio 2015

\section{INTRODUCCIÓN}

En este trabajo se analiza un período de 10 años de mediciones trimestrales de la Encuesta Permanente de Hogares Continua (EPH) del INDEC comprendidas entre el tercer trimestre de 2003 y el cuarto de 2013. Dado que estas estimaciones contienen un error estadístico apreciable ${ }^{1}$, el estudio se limita a señalar las principales tendencias en la evolución de las tasas laborales básicas de desocupación, empleo y actividad; no se formulan hipótesis explicativas respecto de variaciones cuantitativas menores, de corto plazo o referidas a los niveles de las variables para los cuales el error muestral resulta necesariamente más elevado.

La tabla 1 presenta las mencionadas tasas del aglomerado Bahía BlancaCerri $(\mathrm{ABBC})$ y las que corresponden a diversas desagregaciones de interés.

Se han tomado los cuartos trimestres de 2003, 2007 y 2013 para delimitar dos grandes períodos de la evolución económica nacional; el lustro 2003/7 durante el cual la economía nacional salió de la larga crisis iniciada en 1998 y el período 2008/2013, en que la mayor parte de los indicadores económicos, laborales y sociales dejó de mostrar progresos significativos.

El crecimiento del PBI per cápita se redujo de 7,8\% anual en 2003/7 a 2\% en 2008/13 y la desocupación, que en el primer período de cuatro años disminuyó en 6 puntos porcentuales (pp) sólo bajó otros 2 pp en el sexenio 2008/13. Nuestro

* Departamento de Economía (UNS) - Instituto de Investigaciones Económicas y Sociales del Sur (CONICET - UNS). Correo electrónico: burachik@criba.edu.ar.

1 Por ejemplo, el coeficiente de variación de la tasa de desocupación del aglomerado Bahía BlancaCerri en el IV- 2013 fue 16,3\%. Así, los límites del intervalo de $90 \%$ de confianza fueron 6,3 y $11 \%$. 
objetivo es estudiar la evolución de la situación laboral en el ABBC en estas dos etapas y entre los años extremos de la década 2003/13.

Tabla 1. Tasas de actividad, empleo y desocupación. Aglomerado Bahía Blanca-Cerri. Cuarto trimestre de cada año. En \%

\begin{tabular}{|c|c|c|c|c|c|c|c|c|c|}
\hline & \multicolumn{3}{|c|}{$\mathbf{T A}^{1}$} & \multicolumn{3}{|c|}{$\mathbf{T E}^{2}$} & \multicolumn{3}{|c|}{ TD $^{3}$} \\
\hline & 2003 & 2007 & 2013 & 2003 & 2007 & 2013 & 2003 & 2007 & 2013 \\
\hline Aglomerado & 47 & 44 & 48 & 40 & 40 & 44 & 15 & 9 & 9 \\
\hline Varones & 58 & 56 & 58 & 49 & 51 & 56 & 15 & 9 & 5 \\
\hline Mujeres & 39 & 31 & 39 & 33 & 28 & 34 & 15 & 11 & 14 \\
\hline Jefes de hogar & 73 & 69 & 68 & 63 & 63 & 66 & 13 & 9 & 3 \\
\hline Cónyuges & 53 & 39 & 55 & 47 & 36 & 46 & 11 & 6 & 17 \\
\hline Hijos & 24 & 27 & 22 & 19 & 24 & 19 & 22 & 11 & 15 \\
\hline Edad 18-24 & 67 & 47 & 49 & 53 & 45 & 36 & 21 & 174 & 26 \\
\hline Edad 25-30 & 81 & 86 & 85 & 73 & 68 & 75 & 11 & 21 & 11 \\
\hline Edad 31-55 & 83 & 79 & 84 & 74 & 73 & 79 & 11 & 7 & 5 \\
\hline Edad 56-65 & 57 & 53 & 68 & 43 & 45 & 67 & 25 & 15 & 3 \\
\hline N. E. bajo 5 & 45 & 40 & 44 & 36 & 34 & 39 & 21 & 14 & 12 \\
\hline N. E. medio ${ }^{6}$ & 68 & 56 & 59 & 60 & 52 & 55 & 11 & 7 & 7 \\
\hline N. E. superior 7 & 95 & 88 & 84 & 91 & 83 & 78 & 4 & 5 & 7 \\
\hline $\begin{array}{l}\text { Tasa de actividad } \\
2 \text { Tasa de empleo = } \\
3 \text { Tasa de desocupac } \\
\text { El número de deso } \\
\text { En su lugar emple } \\
\text { a altas tasas. } \\
\text { Secundaria incomp } \\
\text { Secundario comple } \\
\text { Estudios superiore }\end{array}$ & $\begin{array}{l}\text { ación/p } \\
=\text { desoc } \\
\text { dos de } \\
\text { s el de I } \\
\text { o meno } \\
\text { estudio } \\
\text { mpletos. }\end{array}$ & $\begin{array}{l}\text { blación } \\
\text { pación/1 } \\
\text { sta edad } \\
-2008, r \\
\text { superio }\end{array}$ & $\begin{array}{l}\text { total. } \\
\text { oblació } \\
\text { en el IV } \\
\text { present }\end{array}$ & $\begin{array}{l}\text { econó } \\
2007 \text { co } \\
\text { tivo del } \\
\text { apletos. }\end{array}$ & $\begin{array}{l}\text { blación } \\
\text { icamen } \\
\text { tenido } \\
\text { período }\end{array}$ & $\begin{array}{l}\text { total. } \\
\text { e activa. } \\
\text { n la base } \\
\text { en que } 1\end{array}$ & $\begin{array}{l}\text { de micr } \\
\text { econom }\end{array}$ & $\begin{array}{l}\text { datos es } \\
\text { ía deja }\end{array}$ & $\begin{array}{l}\text { erróneo. } \\
\text { e crecer }\end{array}$ \\
\hline
\end{tabular}

Fuente: EPH (INDEC). 


\section{EVOLUCION POR PERIODOS}

\section{I.1. Carácter acotado de la recuperación del empleo}

(a) Incidencia de ta y te en la evolución de la td

La disminución de la tasa de desocupación del ABBC de 15\% a 9\% de la población activa constituye un reflejo de la nueva etapa económica que se desarrolló en 2003/7. Como se muestra en la tabla 2, la evolución de la tasa de desocupación de los 31 aglomerados urbanos (31AU) que releva la EPH fue muy similar (de $14 \%$ a $8 \%$ ).

Tabla 2. Tasas de actividad, empleo y desocupación.

Total 31 aglomerados urbanos. Cuarto trimestre de cada año. En \%

\begin{tabular}{|c|c|c|c|c|c|c|c|c|c|}
\hline & \multicolumn{3}{|c|}{ TA } & \multicolumn{3}{|c|}{$\mathrm{TE}$} & \multicolumn{3}{|c|}{ TD } \\
\hline & 2003 & 2007 & 2013 & 2003 & 2007 & 2013 & 2003 & 2007 & 2013 \\
\hline Total aglomerados & 46 & 46 & 46 & 40 & 42 & 43 & 14 & 8 & 6 \\
\hline Varones & 55 & 55 & 54 & 48 & 52 & 51 & 12 & 6 & 6 \\
\hline Mujeres & 38 & 37 & 37 & 32 & 33 & 35 & 17 & 10 & 8 \\
\hline Jefes de hogar & 75 & 73 & 70 & 69 & 69 & 68 & 8 & 4 & 3 \\
\hline Cónyuges & 50 & 50 & 54 & 44 & 47 & 51 & 13 & 7 & 5 \\
\hline Hijos & 28 & 28 & 26 & 21 & 24 & 23 & 27 & 13 & 13 \\
\hline Edad 18-24 & 63 & 58 & 53 & 44 & 48 & 44 & 30 & 16 & 17 \\
\hline Edad 25-30 & 82 & 79 & 79 & 70 & 73 & 74 & 14 & 7 & 7 \\
\hline Edad 31-55 & 81 & 79 & 81 & 73 & 75 & 78 & 9 & 5 & 4 \\
\hline Edad 56-65 & 59 & 59 & 58 & 52 & 56 & 57 & 13 & 5 & 3 \\
\hline
\end{tabular}

Fuente: EPH (INDEC) 
Fue diferente, sin embargo, el proceso subyacente; la caída de la tasa de desocupación local se originó en una contracción de la tasa de actividad con una tasa de empleo constante mientras que a nivel de los $31 \mathrm{AU}$ fue exactamente al revés.

En el segundo subperíodo, entre 2007 y 2013, la tasa de desocupación del $\mathrm{ABBC}$ dejó de disminuir. En estos años la tasa de empleo por fin se incrementó pero el incremento contemporáneo de la tasa de actividad impidió que esto se tradujera en una nueva baja de la tasa de desocupación. A nivel de los 31AU, en cambio, se repitió lo ocurrido en el período anterior (aunque con menor intensidad); nueva disminución de la tasa de desocupación como resultado de un nuevo aumento de la tasa de empleo, con una tasa de actividad constante.

(b) Grado de difusión de la caída de la td y del aumento de la te

Ahora nos interesa verificar el alcance de la disminución de la tasa de desocupación y/o del aumento de la tasa de empleo entre diversos subgrupos de trabajadores. La tabla 1 muestra una desagregación de las tasas básicas según género, edad, rol en el hogar y nivel educativo para el ABBC.

Esta desagregación ofrece elementos de interés para el análisis de la situación de los diversos segmentos que componen a la fuerza de trabajo. Por un lado, permite observar el comportamiento diferenciado de su núcleo central compuesto por los varones adultos "jefes de hogar" respecto de los llamados "trabajadores secundarios", es decir, los cónyuges e hijos. El análisis por tramos de edad, por otro lado, da una pauta del dinamismo del sistema económico para asimilar y brindar oportunidades de ingreso y capacitación a las nuevas generaciones de trabajadores.

Este análisis ofrece un resultado interesante. La caída de la tasa de desocupación fue un fenómeno generalizado en 2003/7 entre los diversos subgrupos de trabajadores. En 2007/13, en cambio, la tasa de desocupación sólo siguió bajando entre los trabajadores varones, jefes de hogar, mayores de 25 años. En otros sectores (mujeres, jóvenes, personas con estudios universitarios completos), por el contrario, volvió a elevarse.

El incremento de la tasa de empleo luego de la larga crisis económica de 1998/2002, en cambio, se fue difundiendo al pasar del primer subperíodo al segundo. En 2003/7 la tasa de empleo sólo aumentó entre los hijos varones mientras que disminuyó entre las mujeres y para los trabajadores menores de 
56 años. En estos últimos sectores la disminución de la tasa de desocupación reposó íntegramente en una disminución de la oferta de trabajadores. En el lustro siguiente, en cambio, el aumento de la tasa de empleo se difundió al conjunto de los sectores de la fuerza de trabajo. Solo que contemporáneamente, como ya se indicó, se produjo un aumento de la tasa de actividad que impidió que la tasa de desocupación continuara bajando.

Resulta de interés la comparación entre esta evolución y la de los 31AU. También en este caso la tasa de desocupación dejó de disminuir al pasar de un período al siguiente en varios sectores (varones, hijos, trabajadores de hasta 30 años). La diferencia residió en el comportamiento de la tasa de empleo; a nivel de los $31 \mathrm{AU}$ en el primer período se incrementó de un modo generalizado mientras que en el segundo sólo siguió aumentando en subsectores específicos (mujeres, cónyuges, mayores de 24) y a contraerse en otros como varones, jefes, hijos de ambos sexos y trabajadores de 18-24 años.

En suma, en el ABBC la disminución de la tasa de desempleo se estancó en 2008/13 porque la tasa de actividad, que se redujo en 2003/7, comenzó a elevarse. A nivel de los $31 \mathrm{AU}$, en cambio, el ritmo de caída de la tasa de desocupación se desaceleró al moderarse y adquirir un carácter más acotado el aumento de la tasa de empleo.

\section{I.2. Obstáculos a la disminución de la desocupación}

Se arriba así a un segundo elemento que surge con claridad de los datos. Se trata de que de aquellas categorías en que la evolución del empleo resultó relativamente desfavorable, emerge una fuerza que mantiene la tasa de desocupación del $\mathrm{ABBC}$ en un nivel elevado tanto en términos históricos ${ }^{2} \operatorname{como}$ respecto a los demás aglomerados urbanos. En el análisis de los factores que influyen sobre la tasa de desocupación resulta útil considerar la siguiente relación entre las tasas laborales básicas ${ }^{3}$ :

$$
\text { Tasa de desocupación } \equiv 1-\frac{\text { Tasa de ocupación }}{\text { Tasa de actividad }}
$$

2 Las mediciones de los primeros dos años de la EPH puntual para el aglomerado ABBC en 1985/7 fluctuaron en \pm 2 pp en torno de una media de $5 \%$.

3 Ver definiciones de estas tasas al pie de la tabla 1. 
Así, la evolución de la tasa de desocupación en un período se puede expresar, alternativamente, en términos de una combinación dada de cambios en las tasas de actividad y empleo. Algunas relaciones son directas e intuitivas (para una tasa de actividad dada, la tasa de desocupación disminuye cuando se reduce la de ocupación). Otras lo son menos; la tasa de desocupación puede subir aunque la tasa de ocupación se incremente, si dicho aumento resulta inferior al de la tasa de actividad.

Por otra parte, al analizar las tasas básicas a nivel de los diversos subgrupos de trabajadores surge una dimensión adicional; aún cuando un subsector dado de la fuerza de trabajo experimente una disminución de su tasa de desocupación seguirá ejerciendo una presión al alza de la tasa del aglomerado si su nivel sigue superando el promedio de este último.

Dos sectores particulares de la fuerza de trabajo, tanto en el ABBC como en los 31AU, muestran una situación laboral relativamente desfavorable; las trabajadoras mujeres y los jóvenes. Son estos segmentos, en ambos casos, los que mantienen la tasa de desocupación en niveles relativamente elevados y son también los que explican la mayor desocupación del ABBC con respecto al total de los aglomerados urbanos del país. En otras palabras, la situación en general desfavorable de mujeres y jóvenes se presenta con particular agudeza en el ABBC.

\section{(a) Mujeres}

En ambos períodos las mujeres mostraron una tasa de desocupación relativamente elevada en el ABBC. En 2003/7 esto se vio reflejado en la alta desocupación de los hijos (fue elevada para los hijos de ambos sexos) y en 2008/13 también en la de los cónyuges ${ }^{4}$.

La desfavorable evolución de la ocupación femenina resulta notable. En 2003/7 la tasa de desocupación de las mujeres se redujo, pero menos que la de los varones. Además, esta reducción no fue el resultado de un aumento de la tasa de empleo (que, de hecho, se contrajo) sino de una brusca disminución de la tasa de actividad. En los años 2008/13, aunque la tasa de empleo de las mujeres experimentó un cierto aumento, la tasa de actividad subió mucho más y por ello la de desocupación no sólo no continuó con el descenso iniciado en los años previos sino que retomó la tendencia ascendente.

4 Otros datos revelan que en el período se aceleró la formación de nuevos hogares de modo que es esperable que el grupo de hijas mujeres desocupadas en 2003/7 figure como cónyuges desocupadas en el período siguiente. 
En el gráfico siguiente, la presión ascendente ejercida por la desocupación femenina se manifiesta a través de valores del índice superiores a la unidad. Como se puede ver, dicha presión estuvo presente de un modo casi permanente, excepto por un breve período en 2010/11.

Dos rasgos parecen caracterizar el comportamiento de la tasa de desocupación femenina del aglomerado. Uno, su nivel fluctúa sistemáticamente por encima de la media. Dos, también sistemáticamente las tasas de ocupación y desocupación femeninas varían en igual sentido debido a las fuertes variaciones de la tasa de actividad; en el primer subperíodo la fuerte contracción de la tasa de actividad permitió que cayera la desocupación pese a la caída de la ocupación y en el siguiente hizo que la tasa de desocupación se incrementara pese al simultáneo incremento de la tasa de ocupación.

Gráfico 1. Tasa de desocupación mujeres/tasa de desocupación varones

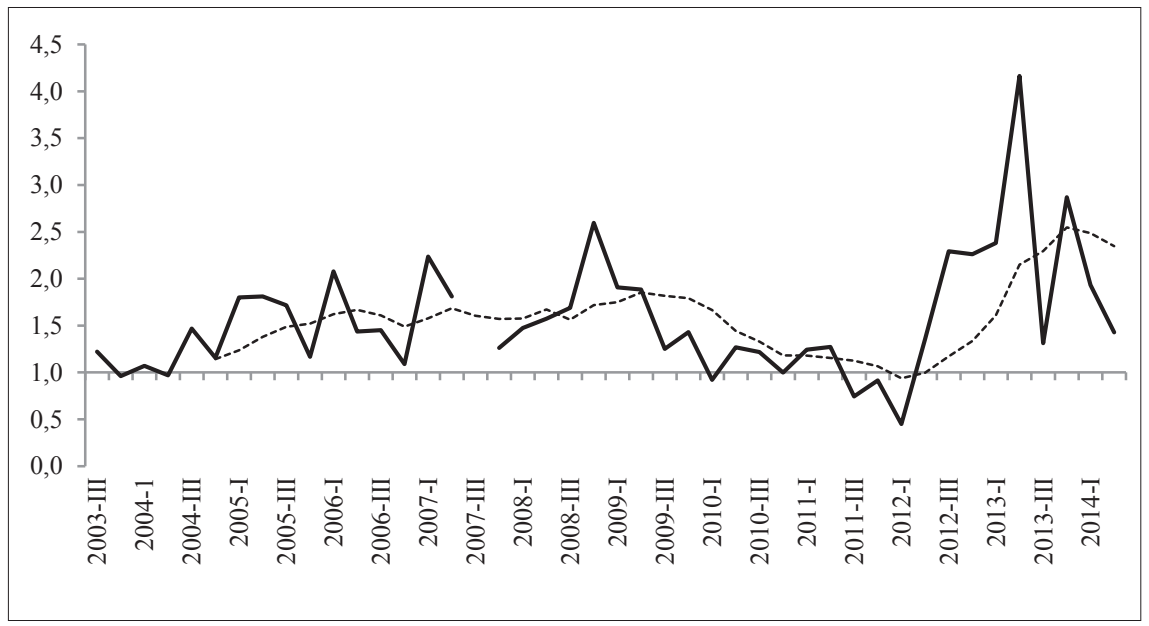

Fuente: EPH (INDEC)

En otras palabras, en 2003/7 muchas mujeres abandonaron o perdieron sus puestos de trabajo y muchas dejaron de buscar; aún así siguió siendo elevada la proporción de las que continuaron solicitando empleo sin encontrarlo. En 2007/13 tuvo lugar un cierto aumento en la disponibilidad de puestos de trabajo para las mujeres pero la cantidad de interesadas que acudió al mercado excedió a las vacantes dando lugar a un nuevo incremento de la tasa de desocupación. La comparecencia masiva de mujeres en busca de empleo (de ingresos adicionales) 
en 2008/13 en un contexto de estancamiento o declinación de los salarios reales contribuyó al deterioro relativo de la situación laboral de las mujeres ya que la tasa de desocupación de los varones continuó disminuyendo.

Hay aquí otro contraste con el total de los aglomerados. Mientras que la tasa de desocupación masculina del $\mathrm{ABBC}$ ha tendido a converger con la de los 31AU, lo contrario ha ocurrido con la femenina que resultó en 2013 6,5 pp (puntos porcentuales) más alta. En IV-2013 la tasa de desocupación de las mujeres bahienses resultó $86 \%$ más alta que en los $31 \mathrm{AU}$.

(b) Jóvenes

También los trabajadores jóvenes muestran una evolución laboral problemática. La tasa de desocupación de los trabajadores de hasta 30 años se ha mantenido sistemáticamente por encima del promedio en el $\mathrm{ABBC}$ al igual que en los 31AU. En ninguno de los dos ámbitos se han notado mejoras sustanciales entre 2008 y 2013.

La elevada tasa de desocupación relativa de los jóvenes se aprecia también cuando se considera el rol en el hogar de los trabajadores; la de los "hijos" (ambos sexos) resulta siempre superior al promedio, tanto en $\mathrm{ABBC}$ como en los $31 \mathrm{AU}$. A decir verdad, la tasa de desocupación de los jefes de hogar del $\mathrm{ABBC}$ ha tendido a converger con su similar de los $31 \mathrm{AU}$; pero las de los demás miembros del hogar (cónyuges e hijos) resultan más elevadas en el ABBC.

En el siguiente gráfico, valores del índice superiores a la unidad muestran niveles de desocupación juvenil más altas que el promedio. Para los dos segmentos de edad seleccionados la tasa de desocupación de los jóvenes resulta sistemáticamente más alta que la de los trabajadores de 31 a 55 años.

En suma, la razón de que la tasa de desocupación del ABBC resulte más elevada que a nivel nacional reside en la mayor desocupación relativa de la fuerza de trabajo "adicional" o "secundaria", es decir, mujeres y jóvenes.

(c) Nivel educativo

Se dispone para el ABBC de información sobre el nivel educativo máximo alcanzado por los trabajadores. La tabla 1 muestra 3 agrupamientos; bajo, medio y alto. Como se observa en la tabla 1, los trabajadores con "bajo" nivel educativo 
presentan sistemáticamente una tasa de desocupación más elevada que la del promedio del $\mathrm{ABBC}$.

En el subperíodo 2003/7, la tasa de desocupación de este grupo de trabajadores se redujo debido a la disminución de su tasa de actividad ya que la tasa de empleo disminuyó. En el subperíodo siguiente la desocupación se mantuvo elevada al producirse una fuerte expansión de la oferta. El gráfico que sigue muestra la elevada tasa de desocupación de los trabajadores de nivel bajo respecto del promedio en la mayor parte de la última década.

Gráfico 2. Tasa de desocupación trabajadores jóvenes/tasa de desocupación trabajadores entre 31 y 55 años

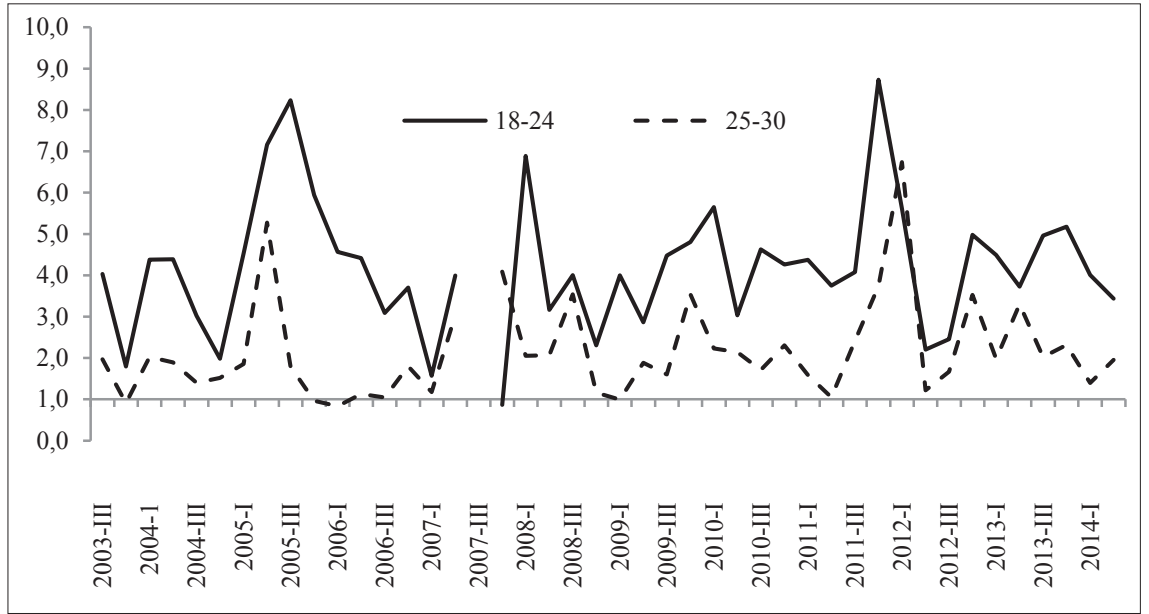

Fuente: EPH (INDEC)

Por último, el gráfico que sigue muestra las diferencias de las tasas de desocupación de la fuerza de trabajo de nivel educativo medio y bajo respecto de las que cuenta con estudios universitarios completos. El gráfico muestra una erosión de la ventaja de inserción laboral vinculada con el nivel educativo. Como se ve, en las primeras mediciones de 2003 la tasa de desocupación de los trabajadores de menor formación era unos 10 puntos porcentuales más alta que la de los universitarios. Esta brecha tendió a estrecharse luego. En los últimos años los trabajadores de nivel educativo "alto" ya sólo muestran una menor desocupación respecto de los trabajadores de nivel educativo bajo; la que tenían hace una década respecto de los de nivel medio parece haber desaparecido. 
Gráfico 3. Tasa de desocupación trabajadores con nivel educativo bajo/tasa de desocupación promedio

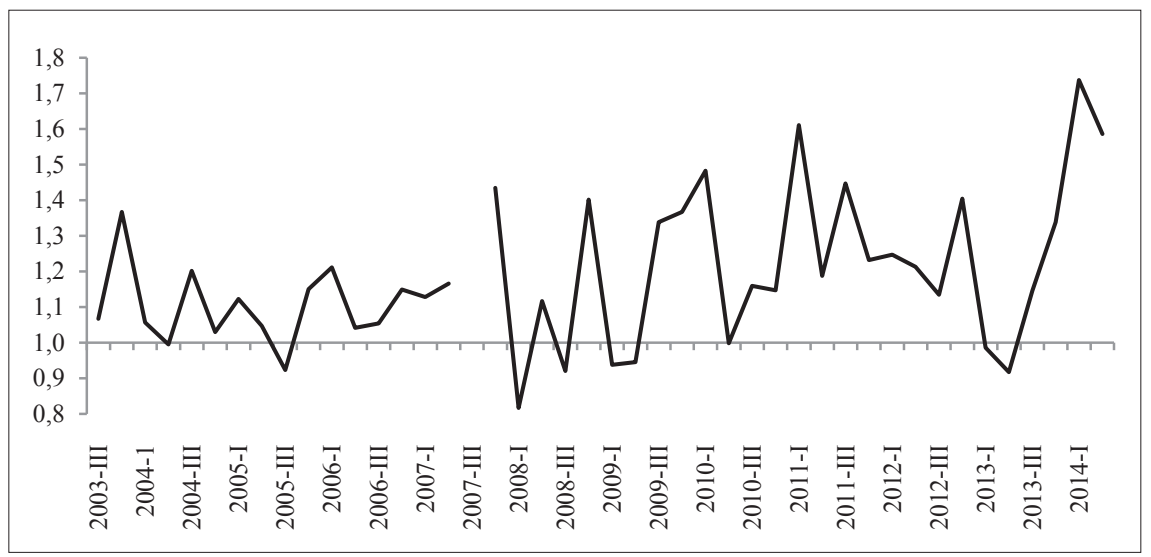

Fuente: EPH (INDEC)

Gráfico 4. Diferencias tasas de desocupación niveles educativos "medio" y "bajo" respecto de "alto" (en puntos porcentuales). Promedio móvil cuatro trimestres

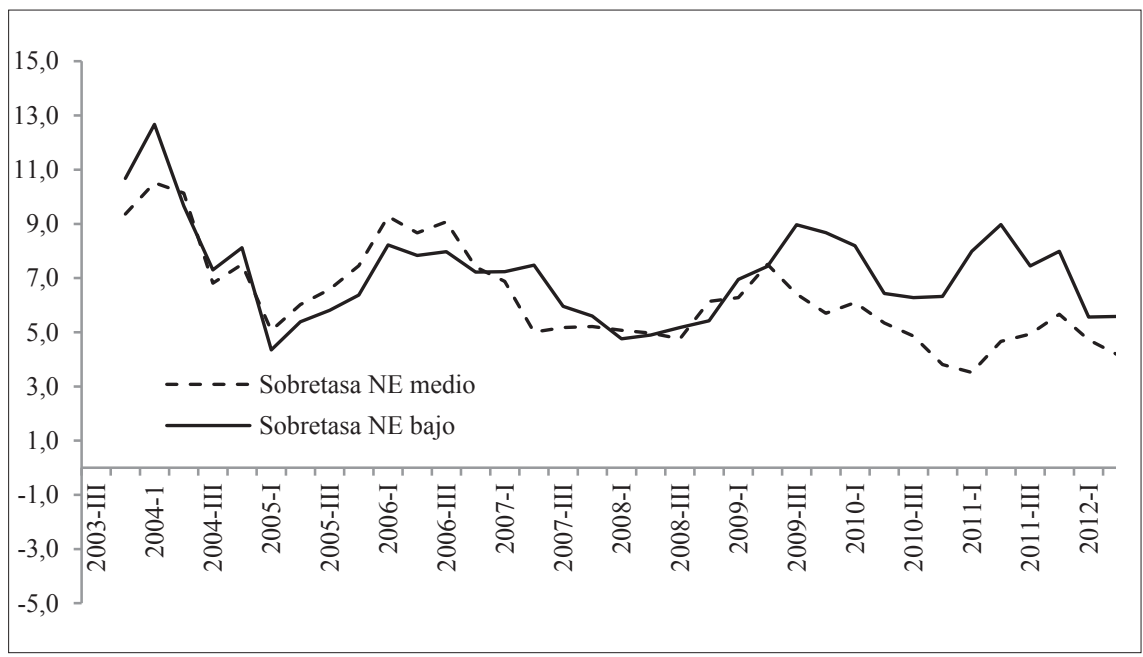

Fuente: EPH (INDEC) 
I.3. Desocupación más elevada que en el resto de los aglomerados urbanos

La tasa de desocupación del ABBC ya era más elevada que en los $31 \mathrm{AU}$ en 2003 y la brecha ha tendido a crecer; en IV-2013 fue 40\% más alta que la del total urbano. Las causas de esta diferencia, además, fueron cambiando. En los primeros años era explicada por la tasa de desocupación relativamente alta de los varones y jefes de hogar pero luego ésta convergió con el promedio urbano nacional y la divergencia pasó a residir en la fuerza de trabajo "secundaria", básicamente mujeres y personas que en el hogar son identificados como cónyuges o hijos. Esto se debió, a su turno, al menor crecimiento de la tasa de empleo de los trabajadores secundarios del $\mathrm{ABBC}$ de la de los $31 \mathrm{AU}$.

También resulta relativamente alta la tasa de desocupación de los trabajadores jóvenes del ABBC. En IV-2013 la tasa de desocupación de los jóvenes bahienses de los segmentos de edad 18-24 y 25-30 resultó un 50\% más alta que en los 31AU. Según los datos esto es explicado, en el caso, de los trabajadores de 18 a 24 años por una particularmente baja tasa de empleo mientras que para los del segmento de 25 a 30 años la explicación reside en una tasa de actividad relativamente elevada.

\section{OCUPACION Y DESOCUPACION A LO LARGO DE LA ULTIMA DECADA}

La tabla 3 ofrece un panorama general de la evolución de los volúmenes de ocupación y desocupación en el $\mathrm{ABBC}$ según las diferentes categorías para el conjunto de la década 2003/13. Se trata de proyecciones poblacionales de los datos muestrales relevados por la EPH. Dado el elevado error característico de estas estimaciones, nos limitamos a señalar sólo algunas tendencias que parecen imponerse con bastante claridad.

El número de ocupados se incrementó $16 \%$ en 10 años, lo que equivale a unos 18.500 puestos de trabajo. La tabla muestra que casi $80 \%$ de dicho incremento correspondió a la fuerza de trabajo masculina.

Además, entre ambos extremos del período, casi toda la creación neta de empleo se focalizó en jefes de hogar con un nivel educativo alto o medio. La composición por edades también se modificó ya que una parte sustancial del incremento neto de la ocupación correspondió a trabajadores mayores de 30 años. Por otra parte, el crecimiento de la ocupación llegó a su punto máximo en el IV- 
2010, es decir, hace más de cuatro años; desde entonces se mantiene estancado en torno de las 134.000 personas.

Respecto de la desocupación pueden señalarse los siguientes elementos. Su disminución en el período 2003 a 2013 fue de 8.000 personas, es decir 38\%. Por otra parte, el agotamiento del proceso de recuperación iniciado en 2003 resultó aún más prematuro que en lo referido a la ocupación. El siguiente gráfico muestra que el mínimo de desocupados se alcanzó en III-2008; desde entonces las personas sin trabajo fluctuaron en torno de 12.500 .

Tabla 3. Ocupados y desocupados. Aglomerado Bahía Blanca-Cerri. Cuarto trimestre de 2003 y de 2013. En miles*

\begin{tabular}{lcccccc}
\hline & \multicolumn{3}{c}{ Ocupados } & \multicolumn{3}{c}{ Desocupados } \\
\hline Aglomerado & 2003 & 2013 & Diferencia & 2003 & 2013 & Diferencia \\
Varones & 117,8 & 136,4 & 18,5 & 20,9 & 12,9 & -8 \\
Mujeres & 67,5 & 81,9 & 14,4 & 12,2 & 4,2 & $-8,1$ \\
Jefes de hogar & 50,3 & 54,5 & 4,1 & 8,7 & 8,8 & 0,1 \\
Cónyuges & 63,1 & 80,9 & 17,8 & 9,8 & 2,1 & $-7,7$ \\
Hijos & 27,9 & 29,5 & 1,6 & 3,6 & 6 & 2,4 \\
Edad 18-24 & 21,2 & 19 & $-2,2$ & 6 & 3,3 & $-2,6$ \\
Edad 25-30 & 20,2 & 13,8 & $-6,4$ & 5,4 & 4,7 & $-0,6$ \\
Edad 31-55 & 18,8 & 26,1 & 7,2 & 2,2 & 3,4 & 1,1 \\
Edad 56-65 & 65,2 & 74,8 & 9,5 & 8,6 & 3,9 & $-4,7$ \\
N. E. bajo & 10,4 & 19,1 & 8,8 & 3,5 & 0,6 & $-2,9$ \\
N. E. medio & 57,8 & 53 & $-4,8$ & 15 & 7 & $-8,1$ \\
N. E. superior & 43,5 & 50 & 6,5 & 5,3 & 3,6 & $-1,7$ \\
\hline
\end{tabular}

* Las sumas de las categorías de rol en el hogar y edad no resultan idénticas a los respectivos totales del aglomerado debido a la omisión de agrupaciones de reducido peso cuantitativo y sin interés para el análisis.

Fuente: EPH (INDEC) 
Gráfico 5. Número de ocupados

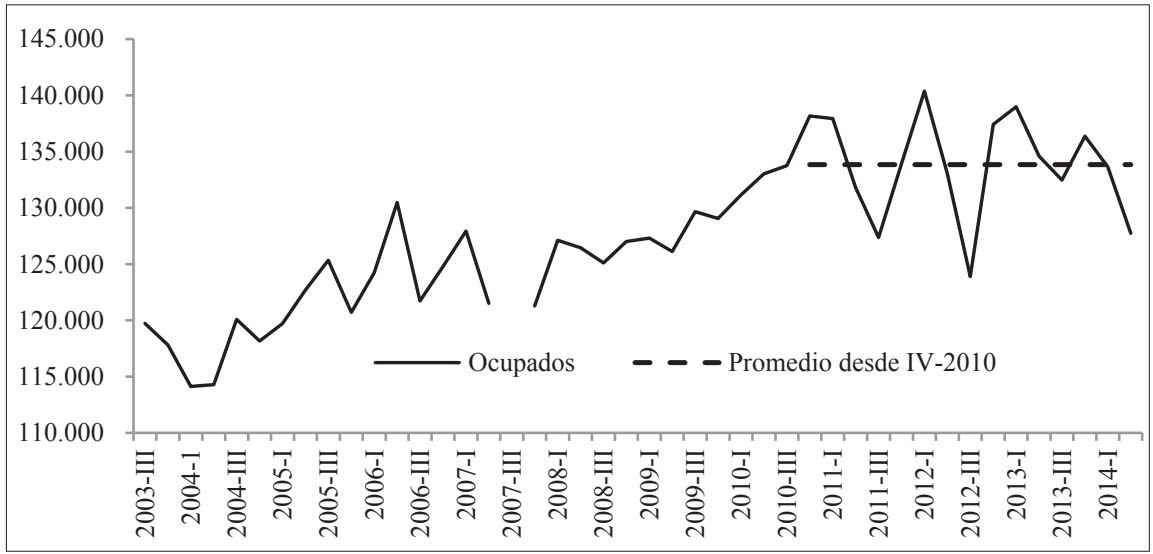

Fuente: EPH (INDEC)

Gráfico 6. Número de desocupados

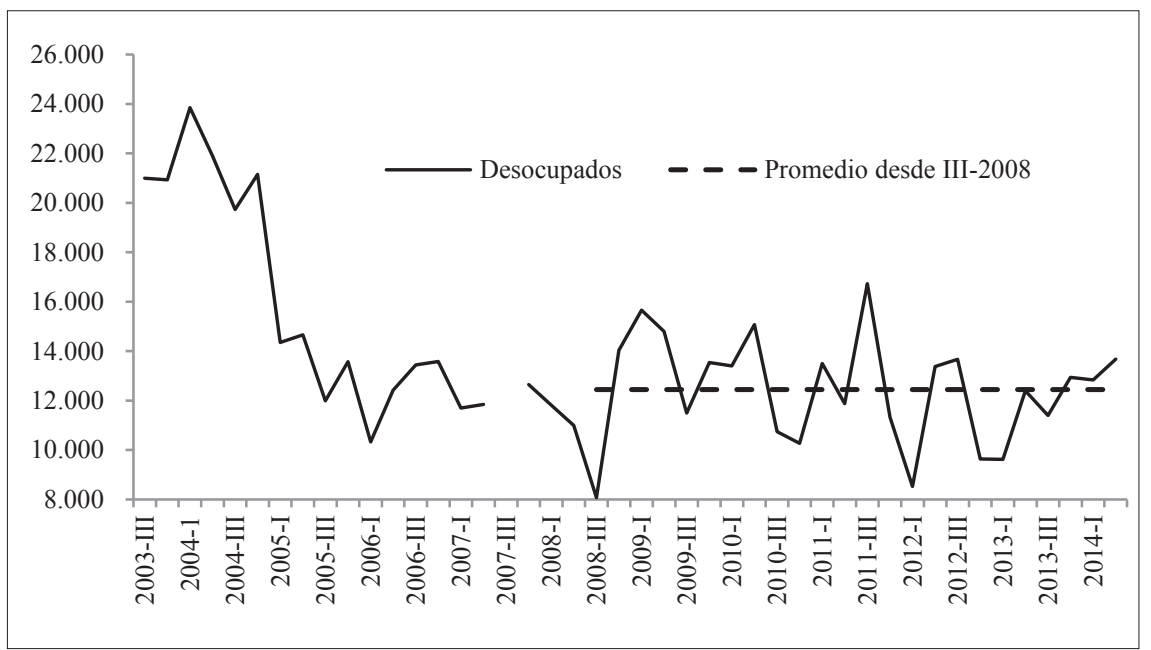

Fuente: EPH (INDEC)

En lo atinente a los diversos componentes de la fuerza de trabajo se pueden comentar algunos elementos de interés. La totalidad de la caída de la desocupación a lo largo de la década 2003/13 correspondió a los varones; el número de mujeres desocupados en 2013 resultó idéntico al de 2003. En 2013 había 67\% más cónyuges 
desocupados que en 2003; como ocurrió con el aumento de la ocupación, la caída de la desocupación se concentró en buena medida en jefes de hogar.

En 2013 había 1.100 jóvenes de 25-30 años desocupados más que en 2003 y el número de desocupados de jóvenes de 18-24 casi no se redujo en toda la década. El grueso de la disminución de la desocupación correspondió a trabajadores de 31-55 y en menor medida a los de 56-65. Por último, el número de desempleados con nivel educativo alto subió en casi 2.000 personas 5 . Curiosamente, se trata del mismo sector laboral que experimentó un gran aumento de los nuevos puestos de trabajo. Los datos muestran que los trabajadores de esta categoría tienen hoy mayor peso en la ocupación pero también en la desocupación, de la que actualmente representan casi un quinto.

El volumen de la fuerza de trabajo con nivel educativo bajo ha disminuido en casi $20 \%$ (entre ocupados y desocupados) a lo largo de la década y esta tendencia seguramente continuará. Con casi la mitad de los desocupados bahienses en 2013 con un nivel educativo medio y alto, los que no alcanzan dicho nivel seguirán sintiendo la presión de terminar los estudios secundarios para elevar sus chances de inserción laboral. Pese a esta tendencia, subsiste un muy voluminoso contingente de trabajadores con nivel educativo "bajo" con una fuerte gravitación en las fluctuaciones de la situación laboral. De hecho en 2003, 72\% de los desocupados del aglomerado tenía nivel educativo "bajo" y en 2007 aún representaba 54\%.

\section{PRECARIZACION LABORAL}

Diversos indicadores referidos a las características de la ocupación permiten entrever dos importantes rasgos de la situación laboral de la última década. El primero se refiere a la dramática profundidad de la crisis laboral generada por los cambios económicos e institucionales introducidos durante los años '90. Como muestra el siguiente cuadro, la precariedad de las relaciones laborales regía para casi la mitad de los ocupados del aglomerado.

El segundo rasgo es que la "reactivación" y "mejoría" de la situación laboral, lejos de restablecer la formalidad en el mercado de trabajo dejó un pesado y sustancial remanente de precariedad; al cabo de una década, se siguen registrando niveles inaceptables de precarización en todos los ítems. Este hecho es particularmente significativo si se considera que varios de los factores que en

5 Debido a la a gran variabilidad que muestra el número de trabajadores en las diversos niveles educativos este dato debe tomarse con cautela. 
la última década favorecieron el crecimiento económico (el colchón de ganancias empresarias generado por la devaluación de 2002, la evolución de los precios mundiales de las materias primas, etc.) han dejado de operar en los últimos años.

Tabla 4. Características de la ocupación principal.

Cuarto trimestre. En \%*

\begin{tabular}{lcc}
\hline & $\mathbf{2 0 0 3}$ & $\mathbf{2 0 1 3}$ \\
\hline Sin vacaciones pagas & 44 & 24 \\
Sin aguinaldo & 44 & 26 \\
Sin días pagos por enfermedad & 48 & 27 \\
Sin obra social & 46 & 27 \\
Sin descuento jubilatorio & 45 & 30 \\
Al cobrar no le dan recibo legal ${ }^{* *}$ & 35 & 28 \\
\hline Promedio simple $^{*}$ & 44 & 27 \\
\hline
\end{tabular}

* Los porcentajes se refieren al subconjunto de los ocupados a los que aplica la pregunta y no al total.

** Incluye a trabajadores que emiten factura.

Fuente: EPH (INDEC)

En el IV-2013 el 27\% indicado en la tabla equivalió a unas 33.000 que se comparan con unas 112.000 respuestas obtenidas para la correspondiente sección del formulario de encuesta de la EPH. Dada la brecha salarial que padecen las personas ocupadas en estas condiciones, es probable que todo o parte del contingente de ocupados precarios integre, junto a los desocupados, el amplio sector de la población del $\mathrm{ABBC}$ con ingresos por debajo de la línea de pobreza.

\section{CONCLUSIONES}

Conviene presentar las conclusiones bajo dos perspectivas diferentes. Por un lado, la evolución de los indicadores laborales del ABBC en la década 2003/13 y, por el otro, la comparación del mercado laboral local con el promedio de los $31 \mathrm{AU}$.

Respecto de la evolución del mercado de trabajo local a lo largo de la década la investigación ha logrado establecer los siguientes hechos salientes:

(a) La tasa de desocupación se redujo en 2003/7 gracias a la disminución de la oferta laboral y no al aumento de la tasa de empleo. Pero esta 
mejoría se agotó prematuramente de modo que la tasa de desocupación se estacionó en un nivel relativamente elevado en términos históricos. El número de desocupados dejó de disminuir en 2008.

(b) Dos sectores laborales muestran una desocupación más alta que la media del aglomerado; la fuerza de trabajo femenina y la de los jóvenes de hasta 30 años. La desocupación local también resulta elevada entre los trabajadores con nivel educativo bajo y en los últimos años se ha observado un deterioro de la situación de los trabajadores con estudios superiores.

(c) La disminución de la tasa de desocupación fue generalizada en 2003/7 porque en los sectores laborales en los que la tasa de empleo no se incrementó se observó una contracción de la tasa de actividad. Pero en el siguiente período (2007/13) sólo siguió bajando para la fuerza de trabajo "central" conformada por los varones adultos jefes de hogar; en los demás sectores la mayor tasa de empleo fue contrarrestada por un incremento de la tasa de actividad.

(d) En el período inmediatamente posterior al estallido de la crisis en 2002, la tasa de desocupación del ABBC tendió a disminuir para los diversos subsectores de la fuerza de trabajo. La tasa de empleo, en cambio, se incrementó para los sectores centrales de la fuerza de trabajo y decreció para los complementarios. Así, la recuperación de los años 2003/7 habilitó la recomposición de los sectores centrales de la fuerza de trabajo en detrimento de los llamados trabajadores "secundarios". Perdieron peso las mujeres, los jóvenes hasta 30 y los cónyuges.

Pero desde 2008 tuvo lugar una reversión de este proceso; la fuerza de trabajo "de reserva" retornó al mercado de trabajo. Esto coincidió con un estancamiento primero y deterioro después del poder adquisitivo de los salarios. Los datos muestran que, si bien se produjo un cierto aumento de la tasa de empleo en 2008/13 este sólo se tradujo en menor tasa de desocupación para los trabajadores centrales. Entre los secundarios, en cambio, o bien la tasa de empleo se mantuvo estable o bien su aumento resultó contrarrestado por el mencionado incremento de la tasa de actividad.

(e) La mayor parte de los puestos de trabajos creados en la década 2003/13 fueron ocupados por varones, jefes de hogar, mayores de 30 años, con nivel educativo intermedio o alto. La situación laboral ha dejado de mejorar hace varios años; la creación de puestos de trabajo alcanzó su pico máximo a fines de 2010 y la disminución de la desocupación se detuvo en el segundo semestre de 2008. 
La evolución del mercado de trabajo del ABBC en la década 2003/13 presenta varias características distintivas respecto del que se observó en el promedio de los aglomerados urbanos.

(a) A diferencia de lo ocurrido en el $\mathrm{ABBC}$, la caída de la tasa de desocupación en los 31AU en los años 2003/7 fue motorizada por el aumento de la tasa de empleo. En el período siguiente la evolución del $\mathrm{ABBC}$ volvió a ser más desfavorable; su tasa de desocupación dejó de disminuir mientras la del promedio urbano nacional continuó bajando, aunque a un menor ritmo que en el período previo.

(b) El ABBC tuvo en toda la década una mayor tasa de desocupación que los 31AU. En 2003/7 esto se debió a los trabajadores "centrales" mientras que en 2007/13 la brecha se debió a la elevada tasa de desocupación de los trabajadores "secundarios".

La investigación arroja dos conclusiones de orden general. Primero, no es posible afirmar que la recomposición de los años posteriores al estallido de 2002 haya revertido los fuertes desequilibrios laborales ocurridos durante la crisis de la convertibilidad en materia de desempleo y precarización. Segundo, al igual que a nivel nacional, el mercado de trabajo urbano bahiense ha dejado de mostrar mejoras significativas en los últimos años.

La superación de estos problemas laborales, tanto a nivel del $\mathrm{ABBC}$ como de los $31 \mathrm{AU}$ en general reclama transformaciones económicas, sociales y políticas de fondo.

\section{REFERENCIAS BIBLIOGRÁFICAS}

INDEC (2003). Encuesta Permanente de Hogares. Recuperado de http://200.51.91.245/argbin/RpWebEngine.exe/PortalAction?\&MODE $=$ MAIN\&BASE $=$ EPH_BASE_FINAL\&MAIN=WebServerMain.inl

INDEC (2007). Encuesta Permanente de Hogares. Recuperado de http://200.51.91.245/argbin/RpWebEngine.exe/PortalAction?\&MODE $=$ MAIN\&BASE $=$ EPH_BASE_FINAL\&MAIN=WebServerMain.inl

INDEC (2013). Encuesta Permanente de Hogares. Recuperado de http://200.51.91.245/argbin/RpWebEngine.exe/PortalAction?\&MODE $=$ MAIN\&BASE $=$ EPH_BASE_FINAL\&MAIN=WebServerMain.inl 
(C) 2015 por los autores; licencia otorgada a la Revista Estudios Económicos. Este artículo es de acceso abierto y distribuido bajo los términos y condiciones de una licencia Atribución-No Comercial 3.0 Unported (CC BY-NC 3.0) de Creative Commons. Para ver una copia de esta licencia, visite http://creativecommons.org/ licenses/by-nc/3.0/ 